\title{
Pain, Depressive Symptoms, and Self-efficacy for Pain Management: Examination in Black Women With Breast Cancer
}

Jennifer Plumb Vilardaga ( $\sim$ jennifer.plumb.vilardaga@duke.edu )

Duke University School of Medicine https://orcid.org/0000-0001-5174-3849

Hannah Fisher

Duke University School of Medicine

Joseph Winger

Duke University School of Medicine

Shannon Miller

Duke University

Christine Nunez

University of Miami School of Medicine

Catherine Majestic

Duke University School of Medicine

\section{Sarah Kelleher}

Duke University School of Medicine

\section{Tamara Somers}

Duke University School of Medicine

\section{Research Article}

Keywords: Breast Cancer, Depression, Health Disparities, Pain, Self-Efficacy

Posted Date: November 29th, 2021

DOI: https://doi.org/10.21203/rs.3.rs-1025255/v1

License: (1) (1) This work is licensed under a Creative Commons Attribution 4.0 International License. Read Full License 


\section{Abstract}

Purpose: Black women with breast cancer face significant disparities, including high levels of pain. Depressive symptoms and self-efficacy for pain management impact how women with breast cancer manage pain, yet little is known about how these variables relate to pain specifically for Black women with breast cancer.

Methods: Baseline regression analyses were conducted using a sample of women $(n=98)$ with stage I-III breast cancer identifying as Black or African-American who were part of a larger intervention trial. Model 1 explored depressive symptoms and pain (i.e., severity and interference). Model 2 explored self-efficacy for pain management and pain. Covariates were age $(M=57.22, S D=10.76)$, cancer stage ( $50 \%=$ stage 1$)$, and education level (36\%=some college).

Results: Participants reported moderate levels of pain severity and interference. Higher depressive symptoms were related to both higher pain severity and interference; $(B=.06, p<.01,95 \% \mathrm{Cl}[.02, .09]$, $\beta=.32)$ and $(B=.13, p<.001,95 \% \mathrm{Cl}[.09,17], \beta=.55)$ respectively. Likewise, lower self-efficacy for pain management was also related to both higher pain severity and interference; $(B=-.04, p<.001,95 \% \mathrm{Cl}$ $[-.05,-.02], \beta=-.44)$ and $(B=-.06, p<.001,95 \% \mathrm{Cl}[-.08,-.04], \beta=-.53)$ respectively. Women reporting less than a high school diploma endorsed significantly higher pain severity and interference than women reporting some college. Age and cancer stage were not significantly related to pain.

Conclusion: Pain for Black women with breast cancer may be influenced by depressive symptoms and self-efficacy for pain management, in addition to other important variables. Attending to better assessment and treatment of depressive symptoms and self-efficacy for pain management may improve outcomes.

\section{Introduction}

Breast cancer is one of the most common cancers for women, affecting approximately 4 million women in the United States [1]. Black women with breast cancer have higher rates of mortality and worse survivorship outcomes than other racial and ethnic groups [2-4]. Black women diagnosed with breast cancer are also more likely to experience higher pain levels compared to other groups [5]. Several factors contribute to these persistent and significant disparities.

Pain related to cancer or its treatment affects more than half of women with breast cancer [6] and is consistently related to poor outcomes for survivors [7]. Black women may carry additional risk for pain above that of non-Hispanic Whites due to both systemic and health risk factors [5, 8]. Systemic factors such as inequitable access to cancer screenings and timely cancer care often mean that Black women may be diagnosed at later cancer stages [9] or require more aggressive treatments [10], contributing to greater pain [7]. Black women with breast cancer may also experience suboptimal pain management related to inadequate screening, concern about medication side effects, fear of addiction, and lack of access to behavioral pain treatments $[3,11]$. Research highlights that Black women are more likely than 
White women to have health risk factors such as being diagnosed at a younger age [9] and with more aggressive cancers (e.g., triple negative breast cancer [TNBC 12]) and these factors are associated with high pain levels [4]. In addition to these health risk factors, there may be other variables relevant to the pain experience of Black women with breast cancer. It is critical to better understand the experience of pain for this group to optimize treatment and improve outcomes.

Black women with breast cancer often endorse depressive symptoms, and depressive symptoms are related to higher pain levels [13]. Changes in physical appearance and functioning due to surgery and other treatments, and concerns about mortality may contribute to depressive symptoms for women with breast cancer [13]. Black women may be at additional risk for depressive symptoms due to systemic stressors such as mistrust in the medical system [14] as well as younger age and later stage at time of diagnosis $[15,16]$. Understanding the burden of depressive symptoms for Black women with breast cancer is important for pain management $[17,18]$.

Self-efficacy for pain management, or one's confidence in her ability to enact particular pain coping strategies, has implications for how women with breast cancer manage pain [19]. In heterogenous samples of cancer patients, self-efficacy for pain management has been shown to help reduce pain and other important pain-related variables (e.g., depression, disability) [20-22]. Black women with breast cancer may be particularly likely to have lower levels of self-efficacy for managing cancer-related pain given the unique stressors they face. Emerging evidence suggests that self-efficacy is also potentially important for Black women with breast cancer. Sheppard and colleagues [23] reported that low selfefficacy for symptom management was associated with increased anxiety and depression for Black women with breast cancer. Likewise, Watkins and colleagues [24] found lower capacity to cope to be associated with higher psychological distress when Black women were receiving chemotherapy. Yet, despite the potential for Black women to carry a higher burden of pain, no study to date has critically examined the role of self-efficacy for pain management in this group.

This study aimed to examine the relationships between pain (i.e., severity, interference), depressive symptoms, and self-efficacy for pain management in a sample of women with breast cancer who identified as Black $(n=98)$. This was an exploratory, secondary analysis of baseline data from a larger trial $(\mathrm{N}=327)$ that assessed a cognitive behavioral pain management intervention for women with breast cancer and pain [25]. The first hypothesis was that Black women who reported higher levels of depressive symptoms would report higher levels of pain (i.e., severity, interference). The second hypothesis was that Black women who reported lower levels of self-efficacy for pain management would report higher levels of pain (i.e., severity, interference). These relationships were expected to be evident even after controlling for demographic and medical variables (i.e., age, cancer stage, education level [26]) known to be related to pain.

\section{Methods}

\section{Participants}


Women at least 18 years of age were recruited from 2017-2020 at Duke University Medical Center and affiliated cancer clinics. Eligibility criteria included the following: 1) diagnosis of stage I-IIIC breast cancer (initial or recurrence) within the past two years; 2) life expectancy $\geq 12$ months; and 3) pain severity rating $\geq 5$ out of 10 at screening, consistent with moderate-to-severe pain [27]. Exclusion criteria included the following: 1) cognitive impairment; 2) brain metastases; 3 ) presence of a severe psychiatric condition (e.g., psychotic disorder or episode, suicidal intent) that would contraindicate safe participation; or 4) current or past ( $<6$ months) engagement in cognitive behavioral coping skills treatment for cancer pain. The parent study was a randomized trial with ethical approval by the Duke University Institutional Review Board (IRB \#: Pro00070823) and registered on ClinicalTrials.gov (NCT02791646).

\section{Procedures}

Potential study participants were assessed for eligibility by study staff using electronic medical record review and mailed recruitment letters and interested participants engaged in informed consent procedures. Written informed consent for participation in study procedures as well as permission for data publication was obtained from enrolled participants. After enrollment in the parent trial, participants completed an online baseline assessment consisting of self-report questionnaires measuring pain severity, pain interference, depressive symptoms, and self-efficacy for pain management. The current analysis is a secondary analysis of baseline data from the larger clinical trial. Additional trial information and another baseline analysis has been published elsewhere $[25,28]$.

\section{Measures}

Demographic and Medical Characteristics. Data were collected through electronic medical record review and self-report. Demographic information included: age, sex, race and ethnicity, education, and household income. Medical characteristics included breast cancer stage and other cancer specific variables (e.g., initial or recurrent diagnosis, surgeries).

Pain Severity and Interference. Pain was assessed with the Brief Pain Inventory (BPI) [29]. Four pain severity items asked participants to rate their pain at its worst, least, and average over the past week as well as their current pain. Response options ranged from 0 (no pain) to 10 (worst pain imaginable). Severity items were averaged for a composite score (Cronbach's $a=.86$ ). Seven pain interference items asked participants to rate the degree to which, over the past week, pain interfered with daily activities (i.e., general activity, mood, walking ability, normal work [including house work], relations with others, sleep, and enjoyment of life). Response options ranged from 0 (does not interfere) to 10 (completely interferes). Pain interference items were averaged for a composite score (Cronbach's $a=.91$ ).

Depressive Symptoms. Depressive symptoms were assessed with the 20-item Center for Epidemiological Studies Depression Scale (CES-D) [30]. Participants were asked to rate how frequently they experienced various depressive symptoms (e.g., low mood, anhedonia, lack of appetite, difficulty concentrating) over the past week. Response options ranged from 0 (rarely or none of the time, less than one day) to 3 (all of the time, five to 7 days). Items were summed for a total score (Cronbach's $a=.91$ ). 
Self-efficacy for Pain Management. Self-efficacy for pain management was assessed with the five-item Chronic Pain Self-Efficacy Scale [31]. Participants were asked to rate their confidence in their ability to decrease their pain, continue their daily activities, keep pain from interfering with their sleep, make a small-to-moderate reduction in pain using methods other than taking extra medication, and make a large reduction in pain using methods other than taking extra medication. Response options ranged from 10 (very uncertain) to 100 (very certain). The five items were averaged for a composite score (Cronbach's a $=.86)$.

\section{Statistical Methods}

Analyses were conducted using SPSS (version 27). Preliminary descriptive analyses included identification of outliers and examination of main study variable distributions for kurtosis, skewness, and assumptions of normality for multivariate data. There were no outliers and all main study variables exhibited normal distributions, with kurtosis and skewness values within -2 and +2 [32]. Bivariate correlations were run for all main study variables: pain severity, pain interference, depressive symptoms, and self-efficacy for pain management. Four regression models were run. Two regression analyses were conducted to examine the association between depressive symptoms and pain severity and pain interference. Likewise, two regression analyses were conducted to examine the association between selfefficacy for pain management and pain severity and pain interference. Regressions were run both with and without covariates (i.e., age, cancer stage, education level). Cancer stage was dummy coded with stage 1 disease as the referent group. Education level was also dummy coded with "some college" as the referent group. Referent groups were determined based on largest represented cancer stage and education level group among our sample. The data that support the findings of this study are available from the corresponding author upon reasonable request.

\section{Results}

\section{Participant Characteristics}

From the parent sample of 327 women with breast cancer, 98 participants (30.0\%) self-identified as Black or African-American. The number of women who identified as Black or African-American enrolled in the parent study was representative of the population served by the treating cancer clinics based on clinic catchment area statistics. The current study used this sub-sample of women identifying as Black or African-American for the analyses. The average age of women in this sub-sample was $57.22(S D=10.87)$ years. Approximately one-third (36\%) of participants reported "at least some college"; nearly half (44\%) of participants reported household income of up to $\$ 39,000 /$ year. For nearly all women (97\%), this was their first breast cancer diagnosis. Forty-nine participants (50\%) had stage 1 disease. Additional demographic and medical characteristics are reported in Tables 1 and 2, respectively. Participant reported pain severity $(M=4.59 ; S D=1.90)$ and interference $(M=4.52 ; S D=2.59)$ were in the moderate range. Additional variable means and correlations are reported in Table 3. 
Table 1

Demographic Characteristics ( $\mathrm{N}=98$ )

\begin{tabular}{|c|c|c|}
\hline & $\mathbf{N}(\%)$ & $M(S D)$ \\
\hline Age (years) & & $57.22(10.78)$ \\
\hline \multicolumn{3}{|l|}{ Race } \\
\hline Black or African-American & $98(100 \%)$ & \\
\hline \multicolumn{3}{|l|}{ Ethnicity } \\
\hline Non-Hispanic & $98(100 \%)$ & \\
\hline \multicolumn{3}{|l|}{ Education } \\
\hline Less than High School Diploma & $5(5.1 \%)$ & \\
\hline High School Diploma & $16(16.3 \%)$ & \\
\hline Some College & $35(35.7 \%)$ & \\
\hline Bachelor's Degree & $24(24.5 \%)$ & \\
\hline Graduate Degree & $18(18.4 \%)$ & \\
\hline \multicolumn{3}{|l|}{ Income } \\
\hline Less than $\$ 10,000$ & $7(7.2 \%)$ & \\
\hline$\$ 10,000$ to $\$ 19,999$ & $14(14.4 \%)$ & \\
\hline$\$ 20,000$ to $\$ 39,999$ & $22(22.7 \%)$ & \\
\hline$\$ 40,000$ to $\$ 59,999$ & $28(28.9 \%)$ & \\
\hline$\$ 60,000$ to $\$ 100,000$ & $18(18.6 \%)$ & \\
\hline More than $\$ 100,000$ & $8(8.2 \%)$ & \\
\hline
\end{tabular}


Table 2

Medical Characteristics ( $\mathrm{N}=98$ )

$\mathrm{N}(\%) \quad M(S D)$

\section{Cancer Diagnosis}

First/Initial

95 (96.9\%)

Recurrence

$3(3.1 \%)$

Months Since Diagnosis

Stage

49 (50.0\%)

II

39 (39.8\%)

III

$10(10.2 \%)$

Mastectomy (one breast only)

Yes

$18(18.8 \%)$

No

$78(81.3 \%)$

Mastectomy (both breasts)

Yes

$12(12.5 \%)$

No

$84(87.5 \%)$

\section{Breast Conserving Surgery}

Yes

$57(59.4 \%)$

No

39 (40.6\%)

Lymph Node Removal

Yes

$15(15.6 \%)$

No

$81(84.4 \%)$

\section{Reconstructive Surgery}

Yes

$13(13.5 \%)$

No

$83(86.5 \%)$

Use of Antidepressant Medication

Yes

$27(27.6 \%)$

Note. $M=$ mean; $S D=$ standard deviation; Breast Conserving Surgery = lumpectomy, quadrantectomy, partial mastectomy, segmental mastectomy. 


\begin{tabular}{|c|c|c|}
\hline & $N(\%)$ & $M(S D)$ \\
\hline No & \multicolumn{2}{|c|}{$71(72.4 \%)$} \\
\hline
\end{tabular}

Table 3

Means $(M)$, Standard Deviations $(S D)$, and Correlation Matrix for Main Study Variables $(\mathrm{N}=98)$

\begin{tabular}{|lllll|}
\hline Variable & $\begin{array}{l}\text { Pain } \\
\text { Severity }\end{array}$ & $\begin{array}{l}\text { Pain } \\
\text { Interference }\end{array}$ & $\begin{array}{l}\text { Pain Self- } \\
\text { Efficacy }\end{array}$ & $\begin{array}{l}\text { Depressive } \\
\text { Symptoms }\end{array}$ \\
\hline M (SD) & $4.59(1.90)$ & $4.52(2.59)$ & $60.14(22.24)$ & $17.20(10.91)$ \\
\hline Pain Severity & 1 & - & - & - \\
\hline Pain Interference & $.73^{\star \star}$ & 1 & - & - \\
\hline Pain Self-Efficacy & $-.50^{\star \star}$ & $-.55^{\star}$ & 1 & - \\
\hline $\begin{array}{l}\text { Depressive } \\
\text { Symptoms }\end{array}$ & $.36^{\star \star}$ & $.59^{\star \star}$ & $-.47^{\star \star}$ & 1 \\
\hline Note. $M=$ mean; $S D=$ standard deviation; Fatigue scores shown as T-score; * $p<.05 ; * \star$ & $p<.01$. \\
\hline
\end{tabular}


Table 4

Predictors of Pain Severity and Pain Interference

Pain Severity

$B \quad p$
Pain Interference

$95 \% \mathrm{Cl}$

$\beta \quad B$

$p$

$95 \% \mathrm{Cl}$

$\beta$

(Lower, Upper

Bounds)
(Lower,

Upper

Bounds)

Model 1

$\begin{array}{lcccccccc}\text { Education Level } & 2.27 & <.01 & .64,3.90 & .27 & 2.34 & <.05 & .39,4.29 & .20 \\ \begin{array}{l}\text { Depressive } \\ \text { Symptoms }\end{array} & .06 & <.001 & .02, .09 & .32 & .13 & <.001 & .09, .17 & .55\end{array}$

(with covariates)

\section{Model 2}

$\begin{array}{lcccccccc}\text { Education Level } & 2.24 & <.01 & .69,3.79 & .26 & 2.30 & <.05 & .26,4.34 & .20 \\ \text { Self-Efficacy } & -.04 & <.001 & -.05,-.02 & -.44 & -.06 & <.001 & -.08,-.04 & -.53 \\ \text { (with covariates) } & & & & & & & & \end{array}$

Note: Models 1 and 2 regression analyses included covariates of age, cancer stage, and education level. Only significant predictors shown. Education Level = less than high school diploma vs. some college.

\section{Relationship between Depressive Symptoms and Pain Severity and Pain Interference}

Without covariates in the regression models, depressive symptoms were significantly related to both pain severity $(B=.06, p<.001,95 \% \mathrm{Cl}[.03,1.00], \beta=.36)$ and pain interference $(B=.14, p<.001,95 \% \mathrm{Cl}[.10, .18], \beta$ $=.59)$, such that higher depressive symptoms were associated with higher pain severity and higher pain interference.

Once covariates were added to the regression models, depressive symptoms remained significantly related to both pain severity $(B=.06, p<.01,95 \% \mathrm{Cl}[.02, .09], \beta=.32)$ and pain interference $(B=.13, p<.001$, $95 \% \mathrm{Cl}[.09, .17], \beta=.55)$. Adding depressive symptoms to the regression models explained an additional $9.0 \%(p<.01)$ and $27.6 \%(p<.001)$ of the variance in pain severity and pain interference, respectively, above and beyond the covariates. The dummy code comparing "less than high school diploma" to "some college" was a significant predictor of both pain severity $(B=2.27, p<.01,95 \% \mathrm{Cl}[.64,3.90], \beta=.27)$ and pain interference $(B=2.34, p<.05,95 \% \mathrm{Cl}[.39,4.29], \beta=.20)$. These results indicate that, on average, women reporting "less than high school diploma" endorsed pain severity and pain interference 2.27 and 2.34 higher than those reporting "some college," respectively. Age and cancer stage were not significantly related to pain. 
Relationship between Self-Efficacy for Pain Management and Pain Severity and Pain Interference

Without covariates in the regression models, self-efficacy for pain management was significantly related to both pain severity $(B=-.04, p<.001,95 \% \mathrm{Cl}[-.06,-.03], \beta=-.50)$ and pain interference $(B=-.06, p<.001,95 \%$ $\mathrm{Cl}[-.08,-.04], \beta=-.55)$, such that lower self-efficacy for pain management was associated with higher pain severity and higher pain interference.

Once covariates were added to the regression models, self-efficacy for pain management remained significantly related to both pain severity $(B=-.04, p<.001,95 \% \mathrm{Cl}[-.05,-.02], \beta=-.44)$ and pain interference $(B=-.06, p<.001,95 \% \mathrm{Cl}[-.08,-.04], \beta=-.53)$. Adding pain self-efficacy to the regression models explained an additional $15.6 \%(p<.001)$ and $21.1 \%(p<.001)$ of the variance in pain severity and pain interference, respectively, above and beyond the covariates. The dummy code comparing "less than high school diploma" to "some college," was a significant predictor of both pain severity $(B=2.24, p<.01,95 \% \mathrm{Cl}[.69$, 3.79], $\beta=.26)$ and pain interference $(B=2.30, p<.05,95 \% \mathrm{Cl}[.26,4.34], \beta=.20)$. These results indicate that, on average, women reporting "less than high school diploma" endorsed pain severity and pain interference 2.24 and 2.30 higher than those reporting "some college," respectively. Age and cancer stage were not significantly related to pain.

\section{Discussion}

The purpose of this study was to examine the relationships between pain, depressive symptoms, and self-efficacy for pain management among women who identify as Black or African American. Higher levels of depressive symptoms were significantly associated with both greater pain severity and pain interference. Lower self-efficacy for pain management was significantly associated with higher pain severity and pain interference. Lower education significantly predicted both greater pain severity and interference in all regression models; age and cancer stage were not independently related to pain. Depressive symptoms and self-efficacy for pain management remained significant predictors of both pain severity and interference over and above the contribution of education level.

The current study is the first to demonstrate that depressive symptoms and self-efficacy for pain management significantly predict pain severity and pain interference, over and above relevant covariates of age, cancer stage, and education level, in a sample of Black women with breast cancer. Literature suggests that this population is particularly vulnerable to high levels of pain due to the influence of numerous health risk and systemic factors. However, this is one of the first studies to examine how psychosocial variables (e.g., depressive symptoms and self-efficacy for pain management) affect the pain experience in this population.

Greater depressive symptoms predicted both higher pain severity and pain interference. Notably, $27 \%$ of the variance in pain interference was explained by depressive symptoms, as opposed to only $9 \%$ of the variance in pain severity. Depressive symptoms such as reduced motivation and persistent negative attitudes about the future may exacerbate systemic vulnerabilities of reduced access to and engagement in care for Black women. When patients experience depressive symptoms alongside greater pain severity 
and even greater pain interference, the combined burden of these symptoms may reduce patients' willingness to seek or persist in accessing adequate cancer care, ultimately worsening outcomes. Indeed, higher overall symptom burden is associated with reduced engagement with breast cancer treatments $[33,34]$. These data lend support for the importance of adequate screening and treatment of depressive symptoms among Black women with breast cancer as a means of improving pain.

While previous studies have examined self-efficacy in relation to anxiety and depressive symptoms for Black women with breast cancer, this is the first study to directly assess the impact of self-efficacy for pain management for this population. We found that lower pain self-efficacy predicted both higher pain severity and higher pain interference. This is consistent with previous work $[20,22,35]$ and provides support for self-efficacy for pain management's relevance for Black women coping with breast cancer and pain. Breast cancer diagnosis and treatment can challenge many women's ability to cope. Black women may be particularly vulnerable to lower self-efficacy for pain management due to health risk and systemic factors. Lack of access to adequate financial and other resources during cancer care, structural racism leading to poor responsiveness from the medical community, and vulnerabilities for more aggressive cancers requiring more intensive treatment may further burden Black women coping with breast cancer. This increased burden may reduce self-efficacy for pain management, leading to greater pain problems because women may not actively seek out and effectively troubleshoot known ways to improve pain (e.g., through pleasant activities, relaxation, exercise).

Pain self-efficacy and depressive symptoms remaining significant predictors of pain over and above the contribution of education level is informative. While education level cannot be changed, self-efficacy for pain management and depressive symptoms can be targeted in evidence-based behavioral interventions (e.g., Pain Coping Skills Training [36]) that teach effective pain coping strategies. In circumstances where breast cancer patients have lower levels of education, targeting pain self-efficacy and depressive symptoms may serve as an effective means of reducing pain. Indeed, promising research shows that lowliteracy adaptations of self-efficacy skills training can improve cancer pain outcomes in medically underserved communities [35].

\section{Future Directions}

If not adequately addressed, cancer-related pain can become persistent pain, leading to poor overall functioning [37]. These results point to the critical importance of adequate screening and treatment of depressive symptoms and pain self-efficacy as a means of treating cancer-related pain and preventing persistent pain among Black women. Larger, longitudinal studies are needed to best characterize these relationships over time. Future research assessing these variables should move beyond those served in a large academic medical center and incorporate those (i.e., in rural areas) who may not have access to such care. Additional variables that can also contribute to disparities in pain outcomes for Black women with cancer, such as patients' experience of racism and medical mistrust [3,38], could be assessed in later studies. Cognitive behavioral interventions promoting self-efficacy have been shown to reduce depressive symptoms, pain severity, and pain interference for breast cancer patients more broadly [22]; 
however, future research should specifically examine and potentially adapt these interventions to best support Black women with breast cancer and pain. Spiritual practices and interpersonal support may also increase self-efficacy [39] and could play an important role in interventions aimed at reducing pain for Black women with breast cancer.

\section{Declarations}

\section{Funding:}

This study was funded through an NIH/NCl 1R01CA202779-01 awarded to senior author, Tamara J. Somers, PhD. The work of Jennifer C. Plumb Vilardaga, PhD was supported in part by a Career Development Award through the Duke University REACH Equity Center; funded by the National Institute on Minority Health and Health Disparities (5U54MD012530-04). The work of Joseph G. Winger, Ph.D. was supported in part by a Kornfeld Scholars Program Award from the National Palliative Care Research Center.

\section{Ethics approval:}

Procedures complied with ethical guidelines and received Duke University Institutional Review Board approval (Pro00070823).

\section{Consent to participate:}

Informed consent was obtained from all individual participants included in this study.

\section{Consent for publication:}

The authors affirm that all human research participants provided informed consent for publication of the data included in this publication.

\section{Conflict of interest/Competing interests:}

All authors declare that they have no financial or other competing interests to disclose.

\section{Availability of data and material:}

$\mathrm{N} / \mathrm{A}$

\section{Code availability:}

N/A

\section{Authors' contributions:}


All authors contributed to the study conception and design. Material preparation, recruitment, delivery of study intervention, and data collection were performed by Jennifer C. Plumb Vilardaga, PhD, Hannah M. Fisher, PhD, Joseph G. Winger, PhD, Shannon N. Miller, BPH, Christine Nuñez, BA, Catherine Majestic, PhD, Sarah A. Kelleher, PhD and Tamara J. Somers, PhD. Data analyses were performed by Hannah M. Fisher, Ph.D. The first draft of the manuscript was written by Jennifer C. Plumb Vilardaga, Ph.D., and all authors commented on subsequent versions of the manuscript. All authors read and approved the final manuscript.

\section{References}

1. DeSantis CE, Ma J, Gaudet MM, et al.: Breast cancer statistics, 2019. CA Cancer J Clin. 2019; 69:438-451.

2. Albano JD, Ward E, Jemal A, et al.: Cancer Mortality in the United States by Education Level and Race. JNCl: Journal of the National Cancer Institute. 2007; 99:1384-1394.

3. Coughlin SS, Yoo W, Whitehead MS, Smith SA: Advancing breast cancer survivorship among AfricanAmerican women. Breast Cancer Res Treat. 2015; 153:253-261.

4. Tammemagi CM: Racial/ethnic disparities in breast and gynecologic cancer treatment and outcomes. Curr Opin Obstet Gynecol. 2007; 19:31-36.

5. Green C, Hart-Johnson T, Loeffler D: Cancer-related chronic pain: examining quality of life in diverse cancer survivors. Cancer. 2011; 117:1994-2003.

6. van den Beuken-van Everdingenvan den M, de Rijke J, Kessels A, Schouten A, van Kleef M, Patijn J: Prevalence of pain in patients with cancer: a systematic review of the past 40 years. Ann Oncol. 2007; 18:1437-1449.

7. Shi Q, Smith TG, Michonski JD, Stein KD, Kaw C, Cleeland CS: Symptom burden in cancer survivors 1 year after diagnosis: a report from the American Cancer Society's Studies of Cancer Survivors. Cancer. 2011; 117:2779-2790.

8. Meghani SH, Polomano RC, Tait RC, Vallerand AH, Anderson KO, Gallagher RM: Advancing a National Agenda to Eliminate Disparities in Pain Care: Directions for Health Policy, Education, Practice, and Research. Pain Medicine. 2012; 13:5-28.

9. Williams F, Thompson E: Disparities in Breast Cancer Stage at Diagnosis: Importance of Race, Poverty, and Age. J Health Dispar Res Pract. 2017; 10:34-45.

10. McGee SA, Durham DD, Tse C-K, Millikan RC: Determinants of breast cancer treatment delay differ for African American and White women. Cancer Epidemiol Biomarkers Prev. 2013; 22:1227-1238.

11. Sun V, Borneman T, Piper B, Koczywas $M$, Ferrell B: Barriers to pain assessment and management in cancer survivorship. J Cancer Surviv. 2008; 2:65-71.

12. Siddharth S, Sharma D: Racial Disparity and Triple-Negative Breast Cancer in African-American Women: A Multifaceted Affair between Obesity, Biology, and Socioeconomic Determinants. Cancers (Basel). 2018; 10:514. 
13. Brandão T, Schulz MS, Matos PM: Psychological adjustment after breast cancer: a systematic review of longitudinal studies. Psychooncology. 2017; 26:917-926.

14. Sheppard VB, Llanos AA, Hurtado-de-Mendoza A, Taylor TR, Adams-Campbell LL: Correlates of depressive symptomatology in African-American breast cancer patients. J Cancer Surviv. 2013; 7:292-299.

15. Costanzo ES, Lutgendorf SK, Mattes ML, et al.: Adjusting to life after treatment: distress and quality of life following treatment for breast cancer. Br J Cancer. 2007; 97:1625-1631.

16. Reyes-Gibby CC, Anderson KO, Morrow PK, Shete S, Hassan S: Depressive symptoms and healthrelated quality of life in breast cancer survivors. J Womens Health (Larchmt). 2012; 21:311-318.

17. Bamonti P, Moye J, Naik A: Pain is associated with continuing depression in cancer survivors. Psychology, Health \& Medicine. 2018; 23:1-14.

18. Shi Q, Michonski J, Stein K, Kaw C, Cleeland C: Symptom burden in cancer survivors 1 year after diagnosis: a report from the American Cancer Society's Studies of Cancer Survivors. Cancer. 2011; 117:2779-2790.

19. White LL, Cohen MZ, Berger AM, Kupzyk KA, Bierman PJ: Self-Efficacy for Management of Symptoms and Symptom Distress in Adults With Cancer: An Integrative Review. Oncol Nurs Forum. 2019; 46:113-128.

20. Kelleher SA, Fisher HM, Winger JG, et al.: Feasibility, engagement, and acceptability of a behavioral pain management intervention for colorectal cancer survivors with pain and psychological distress: data from a pilot randomized controlled trial. Support Care Cancer. 2021; 29:5361-5369.

21. Mosher CE, DuHamel KN, Egert J, Smith MY: Self-efficacy for Coping with Cancer in a Multiethnic Sample of Breast Cancer Patients: Associations with Barriers to Pain Management and Distress. Clin J Pain. 2010; 26:227-234.

22. Somers TJ, Kelleher SA, Westbrook KW, et al.: A Small Randomized Controlled Pilot Trial Comparing Mobile and Traditional Pain Coping Skills Training Protocols for Cancer Patients with Pain. Pain Res Treat. 2016; 2016:2473629.

23. Sheppard VB, Harper FWK, Davis K, Hirpa F, Makambi K: The importance of contextual factors and age in association with anxiety and depression in Black breast cancer patients. Psychooncology. $2014 ; 23: 143-150$.

24. Watkins CC, Kamara Kanu I, Hamilton JB, Kozachik SL, Gaston-Johansson F: Differences in Coping Among African American Women With Breast Cancer and Triple-Negative Breast Cancer. Oncol Nurs Forum. 2017; 44:689-702.

25. Kelleher SA, Dorfman CS, Plumb Vilardaga JC, et al.: Optimizing Delivery of a Behavioral Pain Intervention in Cancer Patients Using a Sequential Multiple Assignment Randomized Trial SMART. Contemp Clin Trials. 2017; 57:51-57.

26. Winther D, Nygaard TK, Horsbø| TA, et al.: Associations between education and physical functioning and pain in adult Danish cancer survivors. Acta Oncologica. 2017; 56:348-353. 
27. Serlin RC, Mendoza TR, Nakamura Y, Edwards KR, Cleeland CS: When is cancer pain mild, moderate or severe? Grading pain severity by its interference with function. Pain. 1995; 61:277-284.

28. Fisher HM, Winger JG, Miller SN, et al.: Relationship between social support, physical symptoms, and depression in women with breast cancer and pain. Support Care Cancer. 2021; 29:5513-5521.

29. Cleeland C, Ryan K: Pain assessment: global use of the Brief Pain Inventory. Rehabilitation Oncology 1995. 1995; 13:29-30.

30. Carleton RN, Thibodeau MA, Teale MJN, et al.: The Center for Epidemiologic Studies Depression Scale: A Review with a Theoretical and Empirical Examination of Item Content and Factor Structure. PLoS One. 2013; 8:e58067.

31. Anderson KO, Dowds BN, Pelletz RE, Edwards TW, Peeters-Asdourian C: Development and initial validation of a scale to measure self-efficacy beliefs in patients with chronic pain. Pain. 1995; 63:77-83.

32. George D, Mallery M: SPSS for Windows Step by Step: A Simple Guide and Reference, 17.0 update. 10th ed. Boston: Pearson, 2010.

33. Yee MK, Sereika SM, Bender CM, Brufsky AM, Connolly MC, Rosenzweig MQ: Symptom incidence, distress, cancer-related distress, and adherence to chemotherapy among African American women with breast cancer. Cancer. 2017; 123:2061-2069.

34. Sitlinger A, Shelby RA, Van Denburg AN, et al.: Higher symptom burden is associated with lower function in women taking adjuvant endocrine therapy for breast cancer. Journal of Geriatric Oncology. 2019; 10:317-321.

35. Dorfman CS, Kelleher SA, Winger JG, et al.: Development and pilot testing of an mHealth behavioral cancer pain protocol for medically underserved communities. J Psychosoc Oncol. 2019; 37:335349 .

36. Keefe FJ, Abernethy AP, C Campbell L: Psychological approaches to understanding and treating disease-related pain. Annu Rev Psychol. 2005; 56:601-630.

37. Andersen KG, Kehlet H: Persistent pain after breast cancer treatment: a critical review of risk factors and strategies for prevention. J Pain. 2011; 12:725-746.

38. Polite BN, Adams-Campbell LL, Brawley OW, et al.: Charting the Future of Cancer Health Disparities Research: A Position Statement From the American Association for Cancer Research, the American Cancer Society, the American Society of Clinical Oncology, and the National Cancer Institute. JCO. 2017; 35:3075-3082.

39. Whitehead NE, Hearn LE: Psychosocial interventions addressing the needs of Black women diagnosed with breast cancer: a review of the current landscape. Psychooncology. 2015; 24:497507. 